\title{
Gemeinsam zu einem gesunden Lebensstil
}

\author{
Die Schweizerische Herzstiftung und die Schweizerische Arbeitsgruppe für kardiale Rehabilitation (SAKR) \\ unterstützen die Gründung von Herzgruppen
}

W. Kottmann*

\footnotetext{
* Präsident der SAKR 2002-2004 Vorsitzender der Herzgruppenkommission der Schweizer Herzstiftung.
}

Korrespondenz:

Dr. med. Wilhard Kottmann Innere Medizin, Kardiologie, Sportmedizin

Rehabilitationszentrum Seewis CH-7212 Seewis
Nach einem akuten kardialen Ereignis ist die konsequente Fortführung einer sekundären Langzeitprävention von entscheidender Bedeutung für den weiteren Verlauf der kardialen Erkrankung.

Im Anschluss an die Behandlung im Akutspital nehmen viele Patienten und Patientinnen an einem Rehabilitationsprogramm teil. Ziel ist es, die kardiale Situation zu stabilisieren, die körperliche Leistungsfähigkeit zu verbessern, die Kenntnis der Risikofaktoren zu vermitteln und den Patienten zu zeigen, was sie selbst zu einem gesünderen Lebensstil beitragen können. Für den Langzeiterfolg der Rehabilitation ist es jedoch wichtig, dass die Betroffenen auch auf Dauer motiviert bleiben, einen gesunden Lebensstil beizubehalten.

Die in der ganzen Schweiz aktiven Herzgruppen wollen helfen, den in der ambulanten oder stationären Rehabilitation erlernten gesunden Lebensstil gemeinsam mit anderen Patienten fortzuführen.

Um in der Schweiz möglichst bald ein flächendeckendes Netz von Herzgruppen zu erhalten, unterstützt die Schweizerische Herzstiftung in Zusammenarbeit mit der SAKR die Gründung und den Aufbau dieser Selbsthilfegruppen.

Dazu wurde jetzt ein Manual erarbeitet, das für interessierte Ärzte und Laien detailliert über die bei der Gründung und Führung einer Herzgruppe notwendigen Schritte Auskunft gibt.

\section{Grundlagen}

Nach einem akuten Herz-Kreislauf-Ereignis sollten die Patientinnen und Patienten in der Regel an einem strukturierten ambulanten oder stationären Rehabilitationsprogramm teilnehmen. Es hilft den Betroffenen, die physischen und psychischen Auswirkungen ihrer Erkrankung besser zu bewältigen, und legt den Grundstein für die den Langzeitverlauf entscheidende Sekundärprävention. Wie wichtig und sinnvoll diese Programme sind, konnte in vielen Studien nachgewiesen werden. Entscheidend ist aber, dass der mit dem Rehabilitationsprogramm gemachte
Anfang seine Fortsetzung im Alltagsleben erfährt. Denn nur eine konsequent durchgeführte Langzeitrehabilitation kann das Risiko eines erneuten Ereignisses, das Risiko von Komplikationen oder das Risiko eines plötzlichen Herztodes nachhaltig und deutlich senken.

Nach WHO-Kriterien unterscheidet man drei Phasen der kardialen Rehabilitation:

- Phase I umfasst die Akutbehandlung während des Spitalaufenthaltes mit der initialen körperlichen Mobilisierung.

- Phase II beinhaltet die ärztlich verordnete ambulante oder stationäre Rehabilitation in einem spezialisierten Rehabilitationszentrum. Sie hat zum Ziel, die grundlegende Information und Motivation zur kardiovaskulären Sekundärprävention zu vermitteln und die für die Betroffenen bestmöglichen physischen, psychischen und sozialen Voraussetzungen für die Rückkehr in das Alltagsund Berufsleben zu schaffen (re-habilitare = wieder fähig machen).

- Phase III umfasst die Langzeitbetreuung der Patienten vorrangig unter der Regie des Hausarztes. Zur Fortsetzung der Bemühungen um eine sekundäre Langzeitprävention sind die Herzgruppen eine für viele Patienten ideale Möglichkeit. Die Mitglieder in der Herzgruppe unterstützen und motivieren sich gegenseitig bei der Bekämpfung von Risikofaktoren und der Umsetzung eines gesunden Lebensstils.

\section{Herzgruppen in der Schweiz}

Im Bewusstsein, dass eine Herzkrankheit oder ein kardiovaskuläres Ereignis nach einer lebenslangen Nachsorge verlangen, entstanden Ende der 70er Jahre die ersten Herzgruppen in der Schweiz. Bei diesen handelte es sich in der Regel um lokale Selbsthilfegruppen, die durch private Initiative von engagierten Ärztinnen/Ärzten und Herzpatienten/-patientinnen entstanden sind, um in ihrer Region ein geeignetes Angebot bereitzustellen. Heute gibt es in der Schweiz bereits mehr als 80 Herzgruppen. Eine Liste mit den 
entsprechenden Adressen kann bei der Schweizerischen Herzstiftung bezogen werden, E-Mail: info@swissheart.ch, oder via Internet www. swissheartgroups.ch.

\section{Was geschieht in einer Herzgruppe?}

Die Aktivitäten von Herzgruppen werden der Phase III der kardialen Rehabilitation zugeordnet. Ziel einer Herzgruppe ist es, durch gemeinsame Aktivitäten die bestehenden kardiovaskulären Risikofaktoren zu korrigieren und zu versuchen, die körperliche Leistungsfähigkeit zu erhalten oder sogar zu verbessern. In der Gruppe kann ein gesundheitsbewusstes Verhalten besser geübt und daher auch leichter in den weiteren Alltag umgesetzt werden.

\section{Bewegungsprogramm zur Verbesserung und Erhaltung der körperlichen Leistungs- fähigkeit}

Das Bewegungstraining ist die Kernaktivität der Herzgruppe. Die Mitglieder treffen sich einbis mehrmals wöchentlich unter der Leitung von speziell ausgebildeten Physiotherapeuten/-therapeutinnen oder Sportlehrer/-lehrerinnen zu einem 60- bis 90minütigem Bewegungsprogramm. Es beinhaltet Gymnastik, Kraftübungen, Ausdauer, Spiel, Entspannungsübungen sowie evtl. auch Aktivitäten im Wasser. Ausdauertraining ist die Basis eines jeden gesundheitsorientierten Trainings. Dynamische Ausdauerbelastungen (z.B. Gehen, Wandern, Joggen, Velofahren, Schwimmen, Skilanglauf) haben sich in vielen Studien als prognostisch besonders günstig für den Verlauf der kardialen Erkrankung erwiesen. Durch ein gezieltes Training kann das Herz-Kreislauf-System ökonomischer arbeiten und wird auf die Dauer belastbarer. Regelmässiges körperliches Training beeinflusst zudem die Risikofaktoren günstig und verbessert das körperliche und psychische Wohlbefinden.

Das Training sollte möglichst frei sein von zu starken Wettkampfanreizen; spielerische körperliche Betätigung und Entspannung stehen im Vordergrund. Es wird ohne Leistungsdruck nach individuellen Möglichkeiten der Patienten trainiert. In vielen Herzgruppen werden auch die oft schon in der Rehabilitationsphase II eingeübten Entspannungsmethoden weiter vermittelt und geübt.

\section{Information, Schulung und Beratung}

Je mehr der Patient über die Funktion des Herzens, mögliche Erkrankungen und deren Ursachen und die Krankheitssymptome weiss, desto besser wird es ihm gelingen, Ängste abzubauen und sich im Alltag wieder sicherer zu fühlen. Nach den grundlegenden Informationen während des Rehabilitationsprogramms der Phase II können die gewonnenen Erkenntnisse durch Wiederholung und Gespräche in der Gruppe verfestigt und so das Beibehalten eines gesunden Lebensstils mit mehr Bewegung und einer gesunderen Ernährung erleichtert werden. Je mehr der Betroffene weiss, desto besser kann er mit seiner Situation umgehen, desto weniger ist er von Fachleuten abhängig bzw. kann diesen gezieltere Fragen zu seiner Situation stellen. Deshalb werden in den Herzgruppen regelmässige Informationstreffen angeboten, in denen erneut aufgezeigt wird, welche Möglichkeiten bestehen, das Risiko des Fortschreitens der Erkrankung und eines erneuten akuten Ereignisses zu verringern.

Es werden alle relevanten Themen wie Bewegungsmangel, Bluthochdruck, erhöhte Blutfettwerte, gesunde Ernährung, Übergewicht usw. nicht nur aus medizinischer Sicht dargestellt, sondern die Betroffenen werden von Fachleuten auch konkret beraten, wie sie ihre individuellen Probleme besser bewältigen können. Die Herzgruppe kann als Gruppe auch bei Raucherentwöhnung sehr gut unterstützend wirken.

\section{Erfahrungsaustausch mit Betroffenen}

Der gegenseitige Austausch unter Betroffenen ist im Vergleich mit einer gewöhnlichen Sportgruppe ein ganz wesentlicher Vorteil der Herzgruppen. Das Gespräch und die gemeinsame Aktivität mit anderen Betroffenen helfen, mit der Krankheit besser umzugehen und notwendige Änderungen des Lebensstils nicht nur als Verzicht, sondern vor allem als Chance zu sehen.

\section{Organisation einer Herzgruppe}

Gemäss der ursprünglichen Idee von Selbsthilfegruppen werden diese oft von Menschen aus direkter persönlicher Betroffenheit gegründet. Viele der Herzgruppen in der Schweiz sind so entstanden. Darüber hinaus haben auch viele Ärztinnen/Ärzte für ihre Patienten die Initiative ergriffen oder andere Fachpersonen wie z.B. Herztherapeuten/-therapeutinnen oder Sportlehrer/-lehrerinnen haben eine Herzgruppe gegründet.

\section{Medizinische Leitung durch einen Arzt oder eine Ärztin}

Zum Start ist die allgemeine Unterstützung durch einen Arzt aus der jeweiligen Region sehr wichtig. Als ärztlicher Herzgruppenleiter über- 
nimmt er unter Berücksichtigung der bestehenden Patienten-Hausarzt-Beziehung die Aufsicht über die Herzgruppe und trägt die Verantwortung für die medizinischen Belange und das Sicherheits- und Notfallkonzept. Er ist fachlich verantwortlich für die Patientenschulung in der Sekundärprävention und zuständig für die Beantwortung medizinischer Fragen der Teilnehmer. Unter Berücksichtigung der PatientenHausarzt-Beziehung hilft er auch bei der Vermittlung von professioneller Unterstützung in therapeutischen Belangen, z. B. Ernährungsberatung, Rauchstopp, Stressmanagement, psychosozialer Betreuung.

\section{Die Herztherapeuten}

Die Leitung des Herz-Kreislauf-Trainings untersteht einem/einer sogenannten Herztherapeuten/-therapeutin. In der Regel übernimmt diese Funktion ein/eine Physiotherapeut/-therapeutin oder ein/eine Sportlehrer/-lehrerin. Ziel ist es, durch ein spezielles Bewegungsprogramm die körperliche Leistungsfähigkeit $\mathrm{zu}$ verbessern. Der/die Herztherapeut/-therapeutin plant und leitet das Herz-Kreislauf-Training und begleitet die Gruppe auch bei anderen sportlichen Aktivitäten. Er/sie ist Verbindungsglied zwischen der medizinischen Leitung und den Mitgliedern und berät bei der Planung weiterer Angebote. Ein Verzeichnis von ausgebildeten Herztherapeuten/-therapeutinnen ist bei der Schweizerischen Herzstiftung zu erhalten.

\section{Rechtliche Form}

In der Schweiz ist die Rechtsform des Vereins für verschiedenste Gruppierungen sehr beliebt. Es ist aber nicht zwingend, dass sich die Herzgruppe als Selbsthilfegruppe diesen rechtlichen Status gibt.

Für die Gründung eines Vereins spricht, dass für administrative, finanzielle und rechtliche Belange klare Strukturen und Regeln das reibungslose Funktionieren einer Gruppe erleichtern. Von der Öffentlichkeit, z.B. den Medien, wird ein Verein meist besser wahrgenommen. Dies hilft unter anderem auch, leichter neue Mitglieder zu gewinnen, oder gegenüber Behörden, die eigenen Interessen besser zu vertreten (z. B. Zurverfügungstellung eines Raumes). Bei der Mittelbeschaffung, z. B. bei Spendengesuchen an das lokale Gewerbe, geniesst ein Verein in der Regel mehr Vertrauen als eine formlose Gruppe.

\section{Wer kann in eine Herzgruppe aufgenommen werden?}

Bei Patienten/-innen, die in einer Herzgruppe aufgenommen werden möchten, sollte der Ge- sundheitszustand während einiger Wochen oder Monaten stabil sein. Dies ist nach dem Durchlaufen einer stationären oder ambulanten Rehabilitation (Phase II) meistens der Fall. Belastungstests am Ende dieser Phase geben Auskunft über die körperliche Leistungsfähigkeit und ermöglichen daher, die Belastbarkeit und die sinnvolle Trainingsintensität in der Herzgruppe zu bestimmen. Wichtig ist die Absprache mit dem Hausarzt und eine Beurteilung des aktuellen Gesundheitszustands durch diesen. Die Schweizerische Herzstiftung stellt ein Anmeldeformular für das Bewegungsprogramm und ein Patientendatenblatt, das vom behandelnden Arzt auszufüllen ist, zur Verfügung.

\section{Anforderungsprofil der SAKR}

Die SAKR bezweckt die Förderung der Beziehungen zwischen den in der Schweiz mit kardiovaskulärer Rehabilitation beschäftigten Personen und Institutionen und legt Wert auf eine qualitativ hochstehende kardiovaskuläre Rehabilitation. Sie hat zu diesem Zweck Anforderungsprofile definiert, denen Rehabilitationsinstitutionen entsprechen müssen, wenn sie von der Arbeitsgruppe offiziell anerkannt sein wollen.

Eine wichtige Bestimmung im Anforderungsprofil für Herzgruppen ist, dass das Programm durch speziell ausgebildete Physiotherapeuten/ -therapeutinnen und Sportlehrer/-lehrerinnen geleitet wird. Für deren Ausbildung organisiert die SAKR jährlich einen Nachdiplomlehrgang Herztherapeut/-therapeutin SAKR. Die Teilnehmerinnen und Teilnehmer können nach dem Lehrgang Herzpatienten/-patientinnen mit verschiedenen Herzerkrankungen in Zusammenarbeit mit Kardiologen und Kardiologinnen selbständig in Rehabilitationsgruppen betreuen. Mit dem bestandenen Abschluss erhalten sie das Diplom Herztherapeut/-therapeutin SAKR. Die Herztherapeuten müssen zudem in CPR ausgebildet sein und jährlich einen Repetitionskurs absolvieren.

Auch für die ärztlichen Herzgruppenleiter/ -leiterinnen bietet die SAKR regelmässig Ausbildungskurse an. Alle zwei Jahre ist die Teilnahme an einem anerkannten Fortbildungskurs obligatorisch. Für die Qualitätskontrolle ist der SAKR jedes Jahr ein Qualitätskontrollformular abzugeben.

\section{Sicherheits- und Notfallkonzept}

Auch wenn alle Statistiken zeigen, dass es während der Bewegungsprogramme nur äusserst selten zu schwerwiegenden Zwischenfällen kommt, muss ein Notfallkonzept vorliegen. Darin muss der Ablauf einer raschen Alarmierung und einer 
professionellen Hilfe durch einen Rettungsdienst festgehalten sein. Das Notfallkonzept ist regelmässig zu überprüfen.

Zudem wird in der Herzgruppe der Gesundheitszustand aller Mitglieder möglichst ausführlich dokumentiert, auch dies dient der Sicherheit des einzelnen Patienten.

\section{Entschädigung durch die Krankenkassen}

Da Herzgruppen eine wichtige Funktion in der Sekundärprävention wahrnehmen, wäre wünschenswert, wenn Krankenkassen Mitgliederbeiträge und weitere Kosten dafür übernehmen würden. Bisher ist dazu die Praxis der Kassen sehr unterschiedlich. Sie variiert zwischen keinen Leistungen und voller Übernahme der Teilnahmekosten in Herzgruppen.

Die Chancen einer Herzgruppe, dass ihre Mitglieder eine finanzielle Unterstützung durch die Krankenkasse erhalten, dürften auch in $\mathrm{Zu}$ kunft grösser sein, wenn die Herzgruppe von einem ausgebildeten Herztherapeuten oder einer -therapeutin geleitet wird bzw. von der SAKR anerkannt ist und deren medizinische Qualitätskriterien erfüllt.

\section{Gründung weiterer Herzgruppen notwendig}

Durch den hohen Kostendruck im Gesundheitswesen sind in den letzten Jahren die Liegezeiten im Akutspital (Phase I) auch bei kardialen Erkrankungen immer mehr verkürzt worden. Dadurch bleibt immer weniger Zeit zur psychischen Ver- arbeitung der Erkrankung und zur Erklärung ihrer Ursachen. Es bleibt zu wenig Zeit, um die Bedeutung der Risikofaktoren zu erklären und das Verständnis für die Notwendigkeit der Sekundärprävention zu wecken.

Um so bedeutender wird die Phase II der Rehabilitation. Hier wird der Grundstein für das Verständnis der Notwendigkeit einer lebenslangen Sekundärprävention gelegt. Neben der weiteren Stabilisierung der kardialen Erkrankungen und der Zeit und Hilfe zur psychischen Verarbeitung ist die Vermittlung von Kenntnissen der Risikofaktoren und des hohen Stellenwertes der Bewegungstherapie von entscheidender Bedeutung.

Nach dieser grundlegenden Motivation in der Phase II der Rehabilitation fragen uns viele Patienten, wie sie jetzt weitere Unterstützung finden können. Häufig können wir ihnen dann eine Herzgruppe empfehlen. Aber nicht überall existiert dieses Angebot. Auch wenn es schon über 80 Herzgruppen in der Schweiz gibt, ist bei weitem noch kein flächendeckendes Netz entstanden. Um möglichst vielen Patienten diese Möglichkeit in annehmbarer Nähe des Wohnortes zu bieten, ist die Gründung weiterer Herzgruppen notwendig. Um zu helfen, den schwierigen ersten Schritt zu machen, hat die Schweizer Herzstiftung in Zusammenarbeit mit der SAKR ein Manual erstellt, das Interessierten die hierzu notwendigen Schritte aufzeigt.

Wir hoffen, dass möglichst viele Laien und Fachleute von diesem Angebot regen Gebrauch machen, damit in Zukunft die meisten Betroffenen eine Herzgruppe in ihrer Nähe finden. 